\title{
Diagnostic Cutoff Value for Ultrasonography of the Common Fibular Neuropathy at the Fibular Head
}

\author{
Ji Yeon Kim, MD, Seojin Song, MD, Hye Jung Park, MD, Won Ihl Rhee, MD, PhD, Sun Jae Won, MD, PhD
}

Department of Rehabilitation Medicine, Yeouido St. Mary's Hospital, College of Medicine, The Catholic University of Korea, Seoul, Korea

\begin{abstract}
Objective To establish the diagnostic cutoff value of ultrasonographic measurement for common fibular neuropathy (CFN) at the fibular head (FH).

Methods Twenty patients with electrodiagnostically diagnosed CFN at the FH and 30 healthy controls were included in the study. The cross-sectional area (CSA) of sciatic nerve at mid-thigh level, common fibular nerve at popliteal fossa (PF), and common fibular (CF) nerve at FH were measured. Additionally, the difference of CF nerve CSA at the FH between symptomatic side and asymptomatic side ( $\triangle \mathrm{Sx}$-Asx), the ratio of CF nerve CSA at FH to at $\mathrm{PF}(\mathrm{FH} / \mathrm{PF})$, and the ratio of CF nerve CSA at the FH symptomatic side to asymptomatic side (Ratio Sx-Asx) were calculated.

Results CSA at the FH, FH/PF, $\triangle \mathrm{Sx}$-Asx, and Ratio Sx-Asx showed significant differences between the patient and control groups. The cutoff value for diagnosing CFN at the FH was $11.7 \mathrm{~mm}^{2}$ for the CSA at the FH (sensitivity $85.0 \%$, specificity $90.0 \%$ ), $1.70 \mathrm{~mm}^{2}$ for the $\Delta \mathrm{Sx}$-Asx (sensitivity $83.3 \%$, specificity $97.0 \%$ ), 1.11 for the $\mathrm{FH} / \mathrm{PF}$ (sensitivity $47.1 \%$, specificity $93.3 \%$ ), and 1.24 for the Ratio Sx-Asx (sensitivity $72.2 \%$, specificity $96.7 \%$ ).

Conclusion The ultrasonographic measurement and cutoff value could be a valuable reference in diagnosing CFN at the FH and improving diagnostic reliability and efficacy.
\end{abstract}

Keywords Ultrasonography, Peroneal nerve, Peroneal neuropathies

\section{INTRODUCTION}

Common fibular neuropathy (CFN) is the most frequent mononeuropathy in the lower extremity [1]. The com- mon fibular nerve, split from sciatic nerve, runs through the popliteal fossa laterally and curves distally around the fibular head (FH) and then divides into superficial and deep branches. The FH is the most common site of lesion

Received November 16, 2015; Accepted April 6, 2016

Corresponding author: Sun Jae Won

Department of Rehabilitation Medicine, Yeouido St. Mary's Hospital, College of Medicine, The Catholic University of Korea, 10 63(yuksam)-ro, Yeongdeungpo-gu, Seoul 07345, Korea. Tel: +82-2-3779-1257, Fax: +82-2-3779-2075, E-mail: gstinfog@catholic.ac.kr

ORCID: Ji Yeon Kim (http://orcid.org/0000-0002-6517-9596); Seojin Song (http://orcid.org/0000-0002-7956-1075); Hye Jung Park (http://orcid. org/0000-0002-6731-4376); Won Ihl Rhee (http://orcid.org/0000-0002-4111-9978); Sun Jae Won (http://orcid.org/0000-0002-9057-3747).

(c) This is an open-access article distributed under the terms of the Creative Commons Attribution Non-Commercial License (http://creativecommons.org/ licenses/by-nc/4.0) which permits unrestricted noncommercial use, distribution, and reproduction in any medium, provided the original work is properly cited. Copyright (C) 2016 by Korean Academy of Rehabilitation Medicine 
in CFN [2]. Main causes of CFN at this level are external compression or direct trauma. Ganglion and systemic disorders such as diabetes mellitus or alcoholism are also known to cause neuropathy $[1,3,4]$. Clinically, patients with CFN at the FH mainly present with foot drop due to weakness of ankle dorsiflexion and paresthesia over lateral calf and dorsum of the foot. However, these symptoms may also be seen in other clinical conditions, including sciatic mononeuropathy, lumbosacral plexopathy, or lumbar radiculopathy $[1,4]$. Therefore, accurate diagnosis and identification of etiology should be made through thorough and straightforward diagnostic measurement. Traditionally, electrophysiologic study has been considered a mainstay in diagnosing peripheral nerve disorder, including CFN at the FH $[4,5]$. Alternatively, ultrasonography a noninvasive means for high-resolution dynamic images in real time has become a preferred tool for assessing peripheral nerves, compensating the limitations of electrophysiologic study such as invasiveness $[1,5,6]$. In addition, ultrasonography shows higher sensitivity, as compared to MRI in noninvasive diagnosis of nerve disorders. The sensitivity of ultrasonography is reportedly $93 \%$, as compared to $67 \%$ for MRI, with similar specificity (86\%) [7].

Nerve enlargement on ultrasonographic measurementis a significant finding in nerve disorders. Measurement of cross-sectional area (CSA) of the nerve is the most widely accepted and reliable method for the diagnosis of nerve disorders $[6,8-10]$. Thus, determination of reference value of CSA is crucial to identification of nerve pathology and proper diagnosis $[6,9]$. However, there are few systemic studies and no standard consensus on ultrasonographic measurements and diagnostic cutoff value for the CFN at the FH [1,6,9-12]. Several studies reported ultrasonographic findings of patients with foot drop and demonstrated the value of ultrasonography as a diagnostic tool; however, they included small number of patients $[1,11,12]$. Moreover, one of the studies on subjects whose etiology was confined to weight loss showed no significant difference in nerve thickening between symptomatic and asymptomatic sides [12]. Recently, >8 $\mathrm{mm}^{2}$ was determined as cutoff value of CF nerve CSA at the FH with a sensitivity of $86 \%$ and a specificity of $73 \%$. Although it was the first report in literature that suggested cutoff value of CF nerve CSA at the $\mathrm{FH}$, the specificity was somewhat low; and there was no comparison of symptomatic and asymptomatic side or that of affected site and nonaffected site at symptomatic side. Also, the 9 subjects with neuropathy other than CFN showed even larger $\mathrm{CF}$ nerve at the $\mathrm{FH}$, as compared to healthy controls, contrary to general expectation [10]. As compared to previous studies, greater number of patients with CFN at the FH with no biased distribution of etiology were included in this study. In addition to absolute nerve CSA, swelling ratio and comparison of symptomatic side and asymptomatic side were also analyzed. Therefore, this study aimed to establish the diagnostic cutoff value of the ultrasonographic measurements, and verify its diagnostic usefulness and efficacy for CFN at the FH.

\section{MATERIALS AND METHODS}

Medical records of 20 patients who visited Yeouido St. Mary's Hospital from January 2013 to April 2014 were reviewed retrospectively; and a control group of 30 healthy age-matched asymptomatic adults were recruited. Ultrasonographic records of right legs were examined prospectively. The study was approved by the Institutional Review Board of Yeouido St. Mary's Hospital. Twenty patients with confirmed CFN at the FH by electrodiagnostic study were included in the patient group. Patients with previous history of trauma or lower leg surgery were excluded. Patients with neurologic disease other than CFN, such as central nervous system disorder, polyneuropathy or combined lumbosacral radiculopathy, plexopathy and sciatic neuropathy were also excluded from the study. Electrodiagnosis was based on recommended guidelines by Preston and Shapiro [13]: (1) slow motor conduction across the FH (delay in the nerve conduction velocity of $>10 \mathrm{~m} / \mathrm{s}$ across the FH), (2) conduction block across the FH (a drop in compound muscle action potential amplitude of $>20 \%$ ), (3) delay of peak latency or a decrease in amplitude in superficial peroneal nerve, and (4) needle electromyograghic abnormalities in at least one peroneal-innervated muscle. Diagnosis of CFN at the FH was confirmed if either (1) or (2) with definite (3), where (4) is considered as an accessory finding [14]. Nerve conduction studies and electromyographic finding of the other nerves, except CF nerve, were required to be normal [10].

Ultrasonography was performed by a physiatrist blinded to the results of clinical and electrodiagnostic study, using a Voluson E ultrasound device (GE Healthcare, 
Waukesha, WI, USA) with a 7-12 MHz linear-array transducer. The physiatrist had more than 3 years of musculoskeletal ultrasonography experience and performed ultrasonography in the outpatient clinic on a daily basis. The probe was carefully placed perpendicular to the nerve to minimize anisotropy and no additional force was applied other than the weight of probe. On transverse scans, the full course of the CF nerve was assessed from the PF to the lateral aspect of the $\mathrm{FH}$, and CSA was measured by continuously tracing inside of hyperechoic epineural rim of the nerve (Fig. 1). During evaluation, both patients and controls were placed in the prone position with knees fully extended. CSA measurements were repeated thrice consecutively and the mean values were obtained. Intra-rater correlation coefficients calculated from control group showed good reliability $(>0.8)$.

CSA was measured at 3 anatomic levels in both groups, as follows: (1) sciatic nerve at mid-thigh level at the site of midpoint of gluteal fold and popliteal crease, (2) CF nerve at the PF just distal to the point of bifurcation from sciatic nerve, and (3) CF nerve at the level of FH before the fibular tunnel. At this site, the largest CSA was recorded. In order to improve accuracy of the ultrasonographic measurement of CFN at the FH [10], in addition to CSA, the following values were calculated: (1) the difference of CF nerve CSA at the FH between symptomatic side and asymptomatic side ( $\triangle \mathrm{Sx}-\mathrm{Asx})(2)$ the ratio of $\mathrm{CF}$ nerve $\mathrm{CSA}$ at the $\mathrm{FH}$ to at the $\mathrm{PF}(\mathrm{FH} / \mathrm{PF})$ (3) the ratio of $\mathrm{CF}$ nerve CSA at the FH symptomatic side to asymptomatic side (Ratio Sx-Asx). In control group, $\Delta S \mathrm{~S}$-Asx and Ratio Sx-Asx were obtained from CSA at the FH of right and left sides.

\section{Statistical analysis}

Statistical analysis was performed with SPSS ver. 17 for Windows (SPSS Inc., Chicago, IL, USA). The chi-square test was used to compare the baseline characteristics of the patients and control groups. The Mann-Whitney U-test was used to analyze differences in CSA measurements and their ratios between the two groups. Threshold of significance was defined as $\mathrm{p}<0.05$, in all tests. A receiver operating characteristic (ROC) analysis was performed to determine optimal cutoff values of the ultrasonographic measurements in diagnosing $\mathrm{CFN}$ at the $\mathrm{FH}$, and specificity and sensitivity were obtained.

\section{RESULTS}

A total of 20 patients and 30 healthy controls were studied. The baseline characteristics showed no significant differences between the two groups ( $p>0.05$ ) (Table 1 ).

Nerve CSA were measured at 3 anatomic levels, as described in Table 2. In patients group, the mean CF nerve CSA at the FH was $14.07 \pm 2.94 \mathrm{~mm}^{2}$ and the difference of CF nerve CSA at the FH between symptomatic side and asymptomatic side $(\triangle \mathrm{Sx}-\mathrm{Asx})$ was $4.83 \pm 2.83 \mathrm{~mm}^{2}$, with significant difference, as compared to control groups ( $p<0.001$ for all). As expected, the mean CSA of sciatic nerve at mid-thigh and that of CF nerve at the PF showed no significant differences between the two groups ( $\mathrm{p}=0.14$ and $\mathrm{p}=0.25$, respectively).

In addition, the FH/PF $(1.27 \pm 0.55)$ and the Ratio Sx-Asx $(1.51 \pm 0.30)$ were significantly larger than that of control group ( $\mathrm{p}=0.04$ and $\mathrm{p}<0.001$, respectively) (Table 2 ).

The diagnostic sensitivity and specificity of ultrasono-
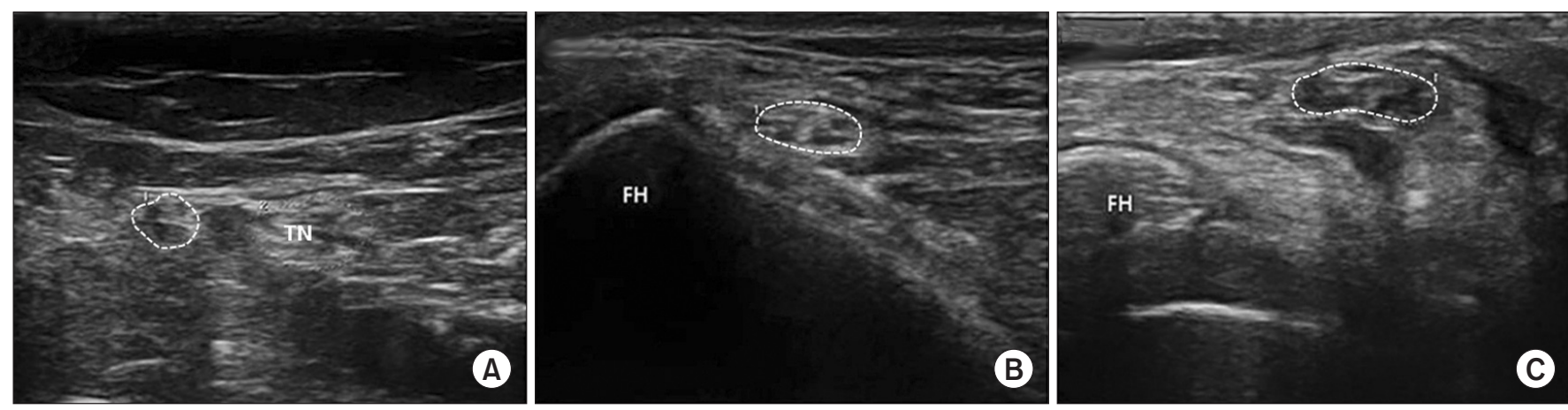

Fig. 1. Ultrasonographic cross-sectional views of the common fibular nerve. Common fibular nerve is shown within dotted line. (A) Common fibular nerve at the popliteal fossa in normal controls, (B) common fibular nerve at the fibular head (FH) in normal controls, and (C) common fibular nerve at the FH in patients. TN, tibial nerve. 
graphic measurements and their ratios were compared by ROC analysis. The cutoff value for the diagnosis of CFN at the FH was $11.70 \mathrm{~mm}^{2}$ for the CF nerve CSA at the FH (sensitivity $85.0 \%$, specificity $90.0 \%$ ), $1.7 \mathrm{~mm}^{2}$ for the $\Delta$ Sx-Asx (sensitivity $83.3 \%$, specificity $96.7 \%$ ), 1.11 for the $\mathrm{FH} / \mathrm{PF}$ (sensitivity $47.1 \%$, specificity $72.2 \%$ ), and 1.24

Table 1. Baseline characteristics of subjects

\begin{tabular}{lcc}
\hline \multicolumn{1}{c}{ Characteristic } & $\begin{array}{c}\text { Control } \\
\text { group } \\
(\mathbf{n = 3 0})\end{array}$ & $\begin{array}{c}\text { Patient } \\
\text { group } \\
(\mathbf{n = 2 0})\end{array}$ \\
\hline Sex (male:female) & $15: 15$ & $16: 4$ \\
\hline Age (yr) & $45.23 \pm 13.58$ & $46.55 \pm 18.52$ \\
\hline Duration of symptom (mo) & - & $6.27 \pm 13.01$ \\
\hline Side (right:left) & $30: 0$ & $9: 11$ \\
\hline Height $(\mathrm{cm})$ & $165.23 \pm 7.24$ & $168.48 \pm 11.41$ \\
Weight $(\mathrm{kg})$ & $63.62 \pm 10.84$ & $68.68 \pm 14.75$ \\
\hline BMI $\left(\mathrm{kg} / \mathrm{m}^{2}\right)$ & $23.24 \pm 3.24$ & $24.01 \pm 3.11$ \\
\hline
\end{tabular}

Values are presented as the number of cases or mean \pm standard deviation. for the Ratio Sx-Asx (sensitivity $72.2 \%$, specificity $96.7 \%$ ), respectively. The $\Delta \mathrm{Sx}$-Asx had the largest area under the curve (AUC, 0.96) (Table 3, Fig. 2).

\section{DISCUSSION}

To date, many studies have reported that ultrasonographic measurement is a beneficial diagnostic tool for nerve disorders with significant positive correlation with electrodiagnostic study [11,15-19]. Ultrasonography visualizes the anatomy of the affected structures and provides morphological information. In some cases, it is crucial for developing therapeutic plans and determining prognosis. In case of CFN at the FH secondary to the intraneural ganglion cyst that needs surgical procedure, ultrasonography leads to more accurate prognosis by detecting the etiology, which might not be identified by electrophysiological study alone [15]. Moreover, if electrodiagnostic study is performed inadequately or insufficiently, ultrasonography could be a supplementary tool for accurate diagnosis by differentiating from lesions that

Table 2. Ultrasonographic measures of the subjects

\begin{tabular}{lccc}
\hline & Control group & Patient group & p-value \\
\hline Sciatic nerve CSA at MT $\left(\mathrm{mm}^{2}\right)$ & $46.56 \pm 6.69$ & $38.33 \pm 10.17$ & 0.14 \\
CF nerve CSA at the PF $\left(\mathrm{mm}^{2}\right)$ & $10.76 \pm 3.47$ & $12.15 \pm 4.28$ & 0.25 \\
CF nerve CSA at the FH-Sx $\left(\mathrm{mm}^{2}\right)$ & $9.32 \pm 2.47^{\mathrm{a})}$ & $14.07 \pm 2.94$ & $<0.001^{*}$ \\
CF nerve CSA at the FH-Asx $\left(\mathrm{mm}^{2}\right)$ & $9.54 \pm 3.10^{\mathrm{b})}$ & $9.83 \pm 2.36$ & 0.46 \\
\hline Sx-Asx at the FH $\left(\mathrm{mm}^{2}\right)$ & $0.22 \pm 1.55$ & $4.83 \pm 2.83$ & $<0.001^{*}$ \\
FH/PF & $0.89 \pm 0.17$ & $1.27 \pm 0.55$ & $0.04^{*}$ \\
Ratio Sx-Asx & $0.99 \pm 0.12$ & $1.51 \pm 0.30$ & $<0.001^{*}$ \\
\hline
\end{tabular}

Values are presented as the number of cases or mean \pm standard deviation.

CSA, cross-sectional area of common fibular nerve; MT, mid-thigh; CF nerve, common fibular nerve; PF, popliteal fossa; $\mathrm{FH} / \mathrm{PF}$, the ratio of CSA of CF nerve at the fibular head to at the PF; $\triangle \mathrm{Sx}$-Asx, difference of CF nerve CSA at the fibular head between symptomatic side and asymptomatic side; ratio Sx-Asx, the ratio of CF nerve CSA at the fibular head symptomatic side to asymptomatic side.

${ }^{*} \mathrm{p}<0.05$, significantly different. ${ }^{\mathrm{a})}$ Right leg of healthy volunteers, ${ }^{\text {b) }}$ left leg of healthy volunteers.

Table 3. Sensitivity and specificity of cutoff values

\begin{tabular}{lcccc}
\hline & Cutoff value & Sensitivity (\%) & Specificity (\%) & AUC \\
\hline CSA at the FH-Sx $\left(\mathrm{mm}^{2}\right)$ & 11.70 & 85.0 & 90.0 & 0.88 \\
$\Delta$ Sx-Asx (FH) $\left(\mathrm{mm}^{2}\right)$ & 1.70 & 83.3 & 96.7 & 0.96 \\
\hline Ratio Sx-Asx (FH) & 1.24 & 72.2 & 96.7 & 0.94 \\
\hline
\end{tabular}

AUC, area under the curve; CSA, cross-sectional area of common fibular nerve; FH, fibular head; $\Delta$ Sx-Asx, difference of common fibular nerve CSA at the fibular head between symptomatic side and asymptomatic side; Ratio Sx-Asx, the ratio of common fibular nerve CSA at the fibular head symptomatic side to asymptomatic side. 


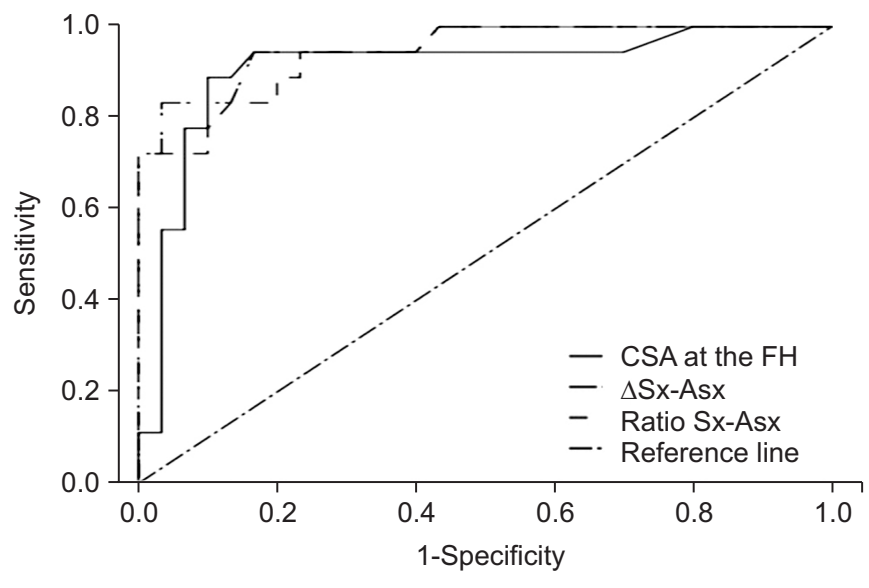

Fig. 2. A receiver operator characteristic (ROC) curve showing the relationship between sensitivity and specificity for each ultrasonographic parameter in diagnosis of common fibular neuropathy at the fibular head. CSA, cross-sectional area of common fibular nerve; FH, fibular head; $\Delta$ Sx-Asx, difference of common fibular nerve CSA at the fibular head between symptomatic side and asymptomatic side; Ratio Sx-Asx, the ratio of common fibular nerve CSA at the fibular head symptomatic side to asymptomatic side.

mimic CFN at the FH such as high sciatic nerve lesion [11]. Ultrasonography in addition to electrodiagnostic study reportedly improves specificity to $88 \%$ in CFN at the $\mathrm{FH}[10]$ and sensitivity up to $98 \%$ in ulnar neuropathy at the elbow [18].

In this study, by conducting ultrasonographic measurement on patients with electrodiagnostically diagnosed CFN at the FH, several cutoff values were confirmed for statistical significance in diagnosing neuropathy.

At the FH, patients had significantly larger mean CSA of CF nerve than controls, consistent with previous studies [10-12]; and cutoff value by the ROC curve was 11.7 $\mathrm{mm}^{2}$, with a sensitivity of $85.0 \%$ and specificity of $90.0 \%$. Mean CSA was $14.07 \mathrm{~mm}^{2}$, which is larger than $10.9 \mathrm{~mm}^{2}$ reported by Visser et al. [10], but smaller than $17.9 \mathrm{~mm}^{2}$ reported by Cruz-Martinez et al. [12], and $26.2 \mathrm{~mm}^{2}$ reported by Lo et al. [11]. Thus, the reported mean CSA had a wide distribution from $11 \mathrm{~mm}^{2}$ to $18 \mathrm{~mm}^{2}$, possibly due to different demographic factors such as height or weight and inclusion of normal healthy subjects alone $[6,10,12,20,21]$.

To overcome these discrepancies, in clinical practice, the healthy contralateral side is often used as an internal control [22-24]. Some previous studies described upper limit value of the side-to-side difference $[6,9,22]$; in addition, one study showed no significant correlation with subjects' baseline demographic factors including age, height, weight, and BMI in CF nerve [9]. Therefore, these absolute values could be a useful reference for detection of nerve pathology in people with different age and body index $[6,9,10,22]$. In our study, mean value of $\Delta S x-A s x$ was $4.83 \pm 2.83 \mathrm{~mm}^{2}$ and $\Delta S \mathrm{~S}$-Asx of $1.7 \mathrm{~mm}^{2}$ as cutoff value yielded a sensitivity of $83.3 \%$ and a specificity $96.7 \%$. In agreement with our findings, previous studies reported $3.2 \mathrm{~mm}^{2}$ and $4.9 \mathrm{~mm}^{2}$ upper limit of side-to-side difference, respectively; but data were obtained from healthy subjects and the validity or diagnostic accuracy were not assessed [6,9].

Although $\Delta$ Sx-Asx was a useful and reliable value, it is not applicable to bilateral lesions or diffuse nerve pathology [22-24]. Therefore, the ratio of nerve CSA has also been analyzed and proposed as a complementary value [25-27]. The swelling ratio of CSA between affected site and unaffected sites is a beneficial method, but most studies are limited to upper extremity nerve disorders, such as carpal tunnel syndrome and ulnar neuropathy at the elbow $[16,23,25,26]$. In this study, ratio Sx-Asx had sensitivity $(72.2 \%)$ and specificity $(96.7 \%)$ at $>1.24$ cutoff value. This value could be helpful, especially in differentiating focal nerve swelling from more diffuse nerve enlargement, such as existing polyneuropathy, which may be due to diabetes mellitus or alcoholism $[10,25,27,28]$.

This study had the limitation of a relatively small number of participants. However, the number of participants in our study exceeded that of previous studies and subjects in patients group had wide variety of etiologies including trauma (30\%), prolonged position or compression (25\%), space occupying lesion around FH (10\%), and metabolic disorders (10\%). Therefore, the result of this study could be used as a generalized standard for patients with CPN at the FH and is considered as representative $[1-4,11]$.

In conclusion, ultrasonographic evaluation of CF nerve and CSA measurement is a helpful and complementary tool for diagnosing CFN at the FH. The diagnostic cutoff value of the ultrasonographic measurements was established and efficacy and validity were verified. Furthermore, this investigation is the first to demonstrate ratio of nerve CSA as useful values, minimizing the effect of demographic factors such as height and BMI. These di- 
agnostic values could facilitate accurate and proper diagnosis of CFN at the FH in clinical practice.

\section{CONFLICT OF INTEREST}

No potential conflict of interest relevant to this article was reported.

\section{REFERENCES}

1. Grant TH, Omar IM, Dumanian GA, Pomeranz CB, Lewis VA. Sonographic evaluation of common peroneal neuropathy in patients with foot drop. J Ultrasound Med 2015;34:705-11.

2. Kim DH, Kline DG. Management and results of peroneal nerve lesions. Neurosurgery 1996;39:312-9.

3. Masakado Y, Kawakami M, Suzuki K, Abe L, Ota T, Kimura A. Clinical neurophysiology in the diagnosis of peroneal nerve palsy. Keio J Med 2008;57:84-9.

4. Van Langenhove M, Pollefliet A, Vanderstraeten G. A retrospective electrodiagnostic evaluation of footdrop in 303 patients. Electromyogr Clin Neurophysiol 1989;29:145-52.

5. Kang S, Kwon HK, Kim KH, Yun HS. Ultrasonography of median nerve and electrophysiologic severity in carpal tunnel syndrome. Ann Rehabil Med 2012;36:729.

6. Cartwright MS, Passmore LV, Yoon JS, Brown ME, Caress JB, Walker FO. Cross-sectional area reference values for nerve ultrasonography. Muscle Nerve 2008; 37:566-71.

7. Zaidman CM, Seelig MJ, Baker JC, Mackinnon SE, Pestronk A. Detection of peripheral nerve pathology: comparison of ultrasound and MRI. Neurology 2013; 80:1634-40.

8. Bargfrede M, Schwennicke A, Tumani H, Reimers CD. Quantitative ultrasonography in focal neuropathies as compared to clinical and EMG findings. Eur J Ultrasound 1999;10:21-9.

9. Seok HY, Jang JH, Won SJ, Yoon JS, Park KS, Kim BJ. Cross-sectional area reference values of nerves in the lower extremities using ultrasonography. Muscle Nerve 2014;50:564-70.

10. Visser LH, Hens V, Soethout M, De Deugd-Maria V, Pijnenburg J, Brekelmans GJ. Diagnostic value of highresolution sonography in common fibular neuropathy at the fibular head. Muscle Nerve 2013;48:171-8.

11. Lo YL, Fook-Chong S, Leoh TH, Dan YF, Tan YE, Lau $\mathrm{WH}$, et al. High-resolution ultrasound as a diagnostic adjunct in common peroneal neuropathy. Arch Neurol 2007;64:1798-800.

12. Cruz-Martinez A, Arpa J, Palau F. Peroneal neuropathy after weight loss. J Peripher Nerv Syst 2000;5:101-5.

13. Preston DC, Shapiro BE. Electromyography and neuromuscular disorders: clinical-electrophysiological correlations. 3rd ed. New York: Elsevier; 2013.

14. Marciniak C, Armon C, Wilson J, Miller R. Practice parameter: utility of electrodiagnostic techniques in evaluating patients with suspected peroneal neuropathy: an evidence-based review. Muscle Nerve 2005; 31:520-7.

15. Visser LH. High-resolution sonography of the common peroneal nerve: detection of intraneural ganglia. Neurology 2006;67:1473-5.

16. Beekman R, Schoemaker MC, Van Der Plas JP, Van Den Berg LH, Franssen H, Wokke JH, et al. Diagnostic value of high-resolution sonography in ulnar neuropathy at the elbow. Neurology 2004;62:767-73.

17. Tsukamoto H, Granata G, Coraci D, Paolasso I, Padua L. Ultrasound and neurophysiological correlation in common fibular nerve conduction block at fibular head. Clin Neurophysiol 2014;125:1491-5.

18. El Miedany YM, Aty SA, Ashour S. Ultrasonography versus nerve conduction study in patients with carpal tunnel syndrome: substantive or complementary tests? Rheumatology (Oxford) 2004;43:887-95.

19. Kerasnoudis A, Tsivgoulis G. Nerve ultrasound in peripheral neuropathies: a review. J Neuroimaging 2015;25:528-38.

20. Gruber H, Peer S, Meirer R, Bodner G. Peroneal nerve palsy associated with knee luxation: evaluation by sonography--initial experiences. AJR Am J Roentgenol 2005;185:1119-25.

21. Peeters EY, Nieboer KH, Osteaux MM. Sonography of the normal ulnar nerve at Guyon's canal and of the common peroneal nerve dorsal to the fibular head. J Clin Ultrasound 2004;32:375-80.

22. Tagliafico A, Cadoni A, Fisci E, Bignotti B, Padua L, Martinoli C. Reliability of side-to-side ultrasound cross-sectional area measurements of lower extremity nerves in healthy subjects. Muscle Nerve 2012;46:71722. 
23. Hobson-Webb LD, Massey JM, Juel VC, Sanders DB. The ultrasonographic wrist-to-forearm median nerve area ratio in carpal tunnel syndrome. Clin Neurophysiol 2008;119:1353-7.

24. Yalcin E, Unlu E, Akyuz M, Karaahmet OZ. Ultrasound diagnosis of ulnar neuropathy: comparison of symptomatic and asymptomatic nerve thickness. J Hand Surg Eur Vol 2014;39:167-71.

25. Bayrak AO, Bayrak IK, Turker H, Elmali M, Nural MS. Ultrasonography in patients with ulnar neuropathy at the elbow: comparison of cross-sectional area and swelling ratio with electrophysiological severity. Muscle Nerve 2010;41:661-6.
26. Fu T, Cao M, Liu F, Zhu J, Ye D, Feng X, et al. Carpal tunnel syndrome assessment with ultrasonography: value of inlet-to-outlet median nerve area ratio in patients versus healthy volunteers. PLoS One 2015;10: e0116777.

27. Yoon JS, Walker FO, Cartwright MS. Ultrasonographic swelling ratio in the diagnosis of ulnar neuropathy at the elbow. Muscle Nerve 2008;38:1231-5.

28. Kerasnoudis A, Pitarokoili K, Behrendt V, Gold R, Yoon MS. Correlation of nerve ultrasound, electrophysiological and clinical findings in chronic inflammatory demyelinating polyneuropathy. J Neuroimaging 2015;25:207-16. 